\title{
Los Sistemas Promediales. Una Articulación Conceptual Entre La Matemática Y La Física
}

\author{
Carlos Rondero-Guerrero, Dr. \\ Arturo Crillo-Pérez, Dr. \\ Roberto Noriega-Papaqui, Dr.
}

Universidad Autónoma del Estado de Hidalgo, México

Doi: 10.19044/esj.2017.v13n36p454 URL:http://dx.doi.org/10.19044/esj.2017.v13n36p454

\begin{abstract}
In this paper we present an alternative analysis of some physical systems through averages. The average systems are defined, which are a clear example of the conceptual articulation between mathematics and physics, which is required to be explicit to foster their learning.
\end{abstract}

Keywords: Average systems, conceptual articulation, mathematics, physics, averages.

\section{Resumen}

En este trabajo se presenta un análisis alterno de algunos sistemas físicos a través de los promedios. Se definen los sistemas promediales, que son una clara muestra de la articulación conceptual entre la matemática y la física, misma que se requiere explicitar para propiciar sus aprendizajes. Particularmente las leyes de la física (conservación del momento lineal y energía) se pueden expresar en términos de promedios.

Palabras claves: Sistemas promediales, articulación conceptual; matemáticas; física; promedios.

\section{Introduction}

En la enseñanza de la física existe una gran cantidad de temas que son tratados por diferentes autores y desde distintas perspectivas, como por ejemplo, Sinarcas y Solbes (2013) han estudiado algunas dificultades de la enseñanza a nivel bachillerato, Sales et all (2015) se han preocupado por la ley de la gravitación universal, otros como Almudi et all (2016), Pocovi y Collivadino (2014) han contribuido a la enseñanza en la teoría de campos y electricidad. Sin embargo, el tema de la presencia de los promedios en la física ha sido poco abordado. Aunque en lo que refiere a la enseñanza de las 
matemáticas, el estudio de los promedios es amplio, Flores (2009), Mederos (2014), entre otros.

En el contexto de este trabajo, consideramos que existen en la física una gran cantidad de sistemas que por sus características son de tipo promedial, en el sentido de ser aquellos donde aparecen en sus desarrollos algunas formas de promedio matemático, como pueden ser, media aritmética, media ponderada, media geométrica o media armónica, además de otros.

Ahora bien, en lo que corresponde a la enseñanza de la física, ocurre frecuentemente que en los libros y manuales que se usan en las asignaturas de bachillerato y nivel universitario, se omite la participación e importancia de los valores promediales en el estudio de ciertos problemas que son prototípicos en el aprendizaje de ésta ciencia. Pareciera entonces, dada la poca presencia de los promedios, que no existe un interés manifiesto, de parte de los autores, por mostrar la trascendencia que tienen en la construcción del conocimiento matemático y físico.

Resulta conveniente remarcar que la presencia de los promedios es fundamental en lo corresponde a su propia estructuración teórica, cuando se estudian en matemáticas los cursos de estadística descriptiva e inferencial, y lo mismo ocurre en el caso específico de asignaturas relacionadas con la física estadística.

Es posible resaltar la importancia de indagar acerca de la necesaria articulación conceptual que existe, entre la matemática y la física, en forma tal que el caso de los sistemas promediales, es una excelente muestra de cómo es que se presenta dicha articulación, en el entendido de que la misma se requiere explicitarla tanto en los manuales como en el aula misma.

Desde una perspectiva histórica, se puede señalar Ortíz (2009), que Arquímedes, fue el primero en poner el énfasis en la articulación conceptual entre la física y la matemática a través de promedios, lo que queda de manifiesto en sus diferentes contribuciones, particularmente en el estudio del equilibrio, en tal caso, en su libro Sobre el equilibrio de la figuras planas, menciona en la Proposición 4:

Si dos magnitudes iguales no tienen el mismo centro de gravedad, el punto medio de la recta que une los centros de gravedad de las magnitudes será el centro de gravedad de la magnitud compuesta de ambas magnitudes.

A lo largo de todo el citado libro y en otras de sus obras, Arquímedes obtiene diferentes resultados sobre le equilibrio de lo planos, pero todo ello está sustentado en la equilibración de los excesos y defectos, cuya relación conceptual directa está dada de una u otra forma, a través de los promedios.

En este trabajo nos restringiremos a presentar y analizar sólo algunos sistemas promediales de la mecánica y el electromagnetismo. En cinemática 
se menciona el teorema de la velocidad media (promedio) o teorema de Merton; en dinámica está el caso de la llamada máquina de Atwood (polea simple sin fricción), en la cual tiene presencia la media armónica de las dos masas que intervienen; en electromagnetismo el caso de resistencias en paralelo y el de capacitores conectados en serie, en ambos aparece también la media armónica de los valores dados. Se presenta además el caso del choque de dos cuerpos, en donde intervienen tanto la media ponderada de las velocidades de ambos cuerpos, además de las medias aritméticas de las velocidades iniciales y finales de cada uno de los dos cuerpos. Finalmente se muestra el problema de los dos cuerpos.

\section{La Presencia De Los Promedios}

En la matemática, la participación de los promedios es de una u otra forma relevante dado que aparecen transversalmente en las diferentes asignaturas, desde la educación básica hasta la universitaria. En los cursos elementales se trabaja por ejemplo, con la media aritmética simple, la cual también tiene presencia en geometría analítica, cuando se requieren calcular las coordenadas del punto medio entre dos puntos dados. En el cálculo diferencial e integral, tienen gran relevancia los llamados teoremas del valor medio para derivadas e integrales. Por su parte, en la estadística descriptiva aparecen la mediana y la moda, además de los diferentes tipos de medias, aritmética, ponderada, geométrica y armónica. Mientras que en la estadística inferencial, los promedios tienen participación en el cálculo de valores esperados (esperanza matemática), varianza, y en los diferentes momentos.

Ahora bien, los promedios posteriormente inciden en estudios diversos de la física estadística, de manera tal que como lo señala (Begg, 2002), desde los aspectos elementales el pensamiento estadístico se puede poner en práctica para a su vez poder sustentar diferentes conceptualizaciones de índole promedial. Lo que a su vez redunda cuando se estudian diversos temas en donde resulta indispensable tratar con los promedios, dada su relevancia en la construcción del conocimiento matemático, lo que a su vez trasciende al conocimiento de la física.

De manera tal que evidenciar la articulación de los saberes matemáticos con los saberes físicos, es un requerimiento obligado para propiciar en los estudiantes aprendizajes con entendimiento que posibiliten el desarrollo de un pensamiento matemático y físico integrado.

Es impotante aclarar que una innovación en el ámbito educativo, como es el caso de incoporar explícitamente los promedios en las asignaturas de física, debe estar sustentada en una conceptualización amplia sobre lo que significa el aprendizaje de cada disciplina y de aquellos aspectos que favorecen la construcción del conocimiento (didácticas específicas). Por supuesto, el aprendizaje de un nuevo saber debe partir del conocimiento 
previo del estudiante; pero frecuentemente este conocimiento viejo se convierte en un obstáculo para la construcción del nuevo conocimiento, por lo que se hace necesario para propiciar la superación de estos obstáculos el incorporar otros significados que son asignados por el nuevo conocimiento (Balacheff, 1990). "Se conoce en contra de un conocimiento anterior, destruyendo conocimientos mal adquiridos... lo que cree saberse claramente ofusca lo que debiera saberse" (Bachelard, 2000, pp. 15-16).

Surgen las preguntas, ¿cómo es que los autores de los libros de física omiten hacer referencia de la presencia e importancia de un determinado valor promedial según sea el problema tratado? ¿qué trascendencia tiene esto en la formación de los estudiantes respecto a sus cursos de física elemental?, ¿acaso no es relevante el comportamiento promedial de los sistemas físicos?

\section{Los Promedios Usuales En La Matemática}

La relación matemática fundamental, conocida por los griegos y usada entre otros aspectos, para calcular raíces, generando sucesiones convergentes de números racionales Rittaud (2009), entre la media armónica $H$, media geométrica $G$ y media aritmética $X$, para dos valores, $x_{1}>0$ y $x_{2}>$ 0 , está dada por la expresión,

$$
\begin{gathered}
H=G^{2} / X . \\
\text { Siendo, } \\
G^{2}=x_{1} x_{2} \quad \text { y } X=\left(x_{1}+x_{2}\right) / 2 .
\end{gathered}
$$

Se cumple además la propiedad conocida para estos tres tipos de promedio,

$$
x_{1} \leq H \leq G \leq X \leq x_{2} .
$$

Particularmente, en el caso en que $x_{1}=x_{2}$, se satisface la igualdad, $H=G=X$. Por otra parte, como es sabido en la matemática hay diferentes tipos de promedios, definidos para una cantidad finita de números. Como una simplificación, particularizamos los casos más conocidos como son las medias aritmética, geométrica, armónica y ponderada, además de las medias cuadrática y potenciada de grado-2, referidas sólo a dos números reales $x, y$. Visto de esta manera, cada una de éstas medias se puede definir como una función (que llamaremos función promedio) $f: R^{2}-R: \quad z=f(x, y)$. Por lo tanto, hacemos referencia a las funciones promedio de la siguiente manera:

1. Media aritmética: $\quad X(x, y)=(x+y) / 2$.

2. Media geométrica: $G(x, y)=(x y)^{1 / 2}$.

3. Media armónica: $\quad H(x, y)=2 x y /(x+y)$.

4. Media ponderada: $M_{p}(x, y ; a, b)=(a x+b y) /(a+b)$.

5. Media cuadrática: $\quad C(x, y)=\left(\left(x^{2}+y^{2}\right) / 2\right)^{1 / 2}$.

6. Media potenciada de grado-2: $M_{2}(x, y)=\left(x^{2}+x y+y^{2}\right) / 3$. 
Es de interés mencionar que la función promedio se puede generalizar a otras tipos de medias como la cuadrática y la potenciada de grado-2.

Dadas las características de las medias anteriores, se presenta la siguiente definición:

Una función promedio $p(x, y)$, asociada a cualquiera de las medias aritmética, geométrica, armónica, cuadrática o potenciada de grado-2, es una función definida en el cuadrante no negativo de $R^{2}$, con valores en los reales, que además cumple con las siguientes propiedades,

1. $p(x, y)=p(y, x)$.

2. $p(1,1)=1$.

3. $p(0,0)=0$, excepto en la media armónica $H(x, y)$.

4. $p(t x, t y)=t^{k} p(x, y)$, función homogénea de grado $k$.

5. $p(x, x)=x^{k}$, función identidad, para homogénea de grado $k$.

Cabe resaltar que a su vez, tales funciones promedio cumplen la propiedad:

$$
x \leq H \leq G \leq X \leq C \leq M_{2} \leq y .
$$

Dicha propiedad queda de manifiesto en las gráficas correspondientes de las tres primeras funciones promedio mencionadas. En la figura siguiente se destaca que la media armónica es la que está por debajo de la media geométrica y la media aritmética. También aparecen los planos $z=x \mathrm{y} z=y$, para fines comparativos.

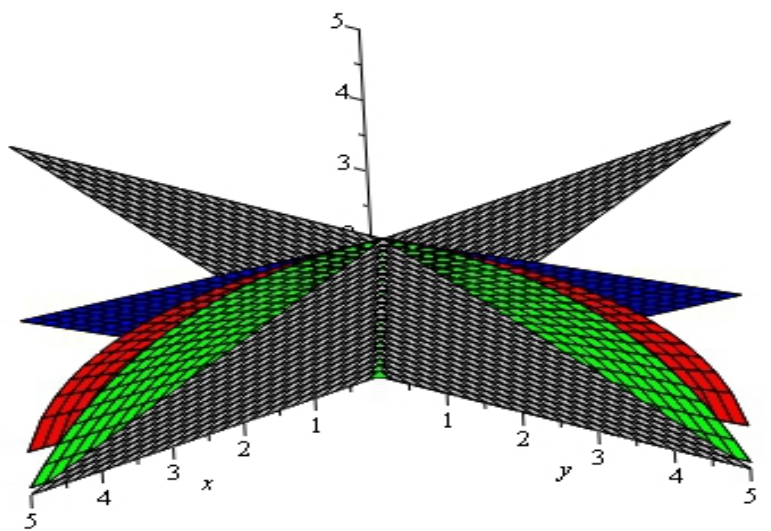

Figura1. Gráfica de funciones promedio 
Las anteriores características y propiedades de las funciones promedio correspondientes, son de considerarse en la enseñanza de la física, ya que al profesor y al estudiante se les provee de las perspectivas analítica y gráfica complementarias en el tratamiento teórico derivado de las medias.

\section{Metodología}

Esta investigación es de corte cualitativo y está sustentada en una aproximación de carácter histórico-epistemológico, que consiste en realizar un rescate de significados inherentes a algunos conceptos matemáticos, que se han ido construyendo en el devenir del tiempo y los cuales son el sustento de la curricula de las asignaturas de matemáticas de bachillerato y nivel superior. Los conceptos fundamentales a los que nos referimos son, las diferentes formas de promedio y el teorema de Pitágoras, entre otros, mismos que permiten identificar ciertos ejes temáticos que posibilitan vislumbrar cómo se presentan diferentes formas de articulación de los saberes matemáticos, en el entendido de que se identifican, ubican y relacionan aspectos semejantes entre conceptos que se complementan unos a otros, pero además permiten dimensionar su trascendencia epistémica y sus incidencias en lo cognitivo y en lo didáctico. Por otra parte, (Rondero, 2015), es de remarcarse que tales conceptos se pueden considerar como referentes epistemológicos (RE), en el sentido de ser conocimiento que tiene una amplia repercusión en el análisis de cómo es que se estructura una red de ideas en torno a los mismos, lo que por otra parte, debiera ser incluido en la formación los profesores de matemáticas y física.. En este trabajo, los promedios matemáticos, se consideran un referente epistemológico (RE), que también se entiende, como una idea o concepto matemático que constituye la base para establecer nuevos conocimientos, o bien, ideas o conceptos que pueden extenderse a otros ámbitos, en este caso en la física, integrando, de esta forma, una red conceptual de significados. Adicionalmente, un referente epistemológico (RE), se puede pensar como el centro de un entramado en el cual se sustenta la estructura de conceptos construida en torno a ese elemento central. $\mathrm{O}$ bien, en otro sentido, el referente epistemológico es de origen un saber sabio a través del cual se va entretejiendo una red de saberes enseñables. En este caso, a través de la media aritmética ponderada, se estructura la red de conceptos en la física tales como: velocidad media; o bien el promedio que se expresa en la resiencia equivalente en un circuito conectado en serie, la velocidad del centro de masa, en la colisión de dos cuerpos, o el promedio de los cuadrados de las velocidades iniciales y finales de ambas partículas. Por su parte, la media armónica, da lugar a una red de conceptos que se pueden identificar como la masa promedio en la tensión de la cuerda de la máquina de Atwood; la capacitancia equivalente en un circuito en serie, o en su caso, la masa reducida que apaece en el problema 
de dos cuerpos. Todos estos tratamientos conforman una red de significados que son propios de la física y le sustento a los sistemas promediales, en la medida de que se va haciendo evidente la partición de los promedios en el estudio de diferentes sistemas físicos. De ése modo, la articulación de saberes matemáticos y físicos se va explicitando y su potencial conceptual se hace necesario para propiciar sus aprendizajes. En tal caso, las diferentes formas de promedio, están articulados conceptualmente con

\section{Sistema físico}

Desde el punto de vista físico, una definición acertada de un sistema físico es la dada por (García-Colín, 1970):

Una porción de universo que se aísla para su estudio.

Así, las partículas contenidas en una gota de agua, abejas en un panal, una fogata, un sistema planetario, un automóvil moviéndose sobre una carretera, son ejemplos de sistemas físicos. Aunado a un sistema físico existen otros dos elementos importantes, los alrededores o entorno y la frontera o pared. Como alrededor se entiende todo aquello que interacciona con el propio sistema físico, la frontera, como lo que separa de los alrededores, el tipo de frontera establece la interacción entre el sistema y los alrededores. Un tipo particular de frontera es aquella en que el sistema no interactúa con los alrededores y es llamada frontera aislante.

Por lo que se refiere al número de elementos que integran al sistema físico, existen dos áreas marcadas en la física, por un lado está la Mecánica Clásica y por otro la Mecánica Estadística, la primera se puede visualizar como la física de un pequeño número de elementos (uno, dos y/o tres) y la segunda como la física de las multitudes $\left(10^{23}\right.$ elementos). En este trabajo nos enfocaremos sólo a sistemas concernientes con pocos elementos.

Una vez caracterizado el sistema a estudiar, es necesario conocer la interacción o relación que guardan los elementos que lo conforman. En la física se trabaja con expresiones analíticas de muy pocas fuerzas de interacción: las leyes de la Gravitación Universal, Hook, Coulomb y de Biot-Savart, son ejemplos de interacciones analíticas conocidas (Giancoli, 2015, Serway \& Jewett, 2014, Halliday, Resnick \& Walker, 2013).

\section{Sistemas Promediales}

En física se sabe que cuando se tienen dos cuerpos cuya interacción solo depende de la distancia relativa, siempre es posible llevarlo a un sistema equivalente de un solo cuerpo, inmerso en un potencial respectivo (Goldstein, 2001). Sin embargo, no es en el único lugar donde aparecen, también los sistemas equivalentes se encuentran en electromagnetisno cuando se estudian las resistencias y los capacitores en serie y en paralelo (Giancoli, 2015, Serway \& Jewett, 2014, Halliday, Resnick \& Walker, 
2013), en mecánica clásica básica, en el choque de dos cuerpos (Giancoli, 2015, Serway \& Jewett, 2014, Halliday, Resnick \& Walker, 2013).

Particularmente, en este trabajo interesa mostrar algunas de las contribuciones que se pueden identificar al estudiar sistemas físicos en donde aparece algún tipo de promedio.

Dentro de una perspectiva sistémica, es conveniente hacer referencia al significado de un sistema físico de tipo promedial. Para tal fin, se requiere acudir a la teoría general de sistemas (Bertalanffy, 1989), la que afirma que las propiedades de los sistemas no pueden describirse significativamente en términos de sus elementos separados. La compresión de los sistemas sólo ocurre cuando se estudian globalmente, involucrando todas las interdependencias de sus partes. Es decir, el funcionamiento de un sistema puede ser descrito por un conjunto de variables interrelacionas.

Por supuesto, un sistema físico se describe por medio de un modelo matemático, en donde aparecen al menos dos cantidades pasivas (masas, resistencias, capacitancias, o bien cantidades activas, (posiciones $\mathrm{o}$ velocidades), cuando es posible reducirlo a un sistema equivalente, en donde aparecen uno o varios promedios (media aritmética, ponderada, armónica geométrica, entre otros), entonces a este sistema equivalente le llamaremos sistema promedial, lo que permite tener una descripción complementaria del sistema original. Por tanto, un sistema promedial en la física, es aquel que para su descripción y análisis, De tal manera, que el comportamiento del sistema no cambia, pero sí su interpretación, cuando se involucra un tipo de promedio conocido, o bien en su caso, se introduce algún otro.

\section{Sistemas Promediales En La Cinemática}

Los sistemas promediales que se muestran a continuación son de tipo determinista, es decir, sus sustentos teóricos, como la leyes de Newton, como se sabe son de esencia determinista, dado que se pueden predecir sus comportamientos en todo tiempo.

Resulta conveniente iniciar el estudio de los sistemas promediales con el movimiento uniformemente acelerado (MUA), dada la importancia didáctica, conceptual y aplicada que tiene en la física elemental.

Como es bien conocido, las ecuaciones básicas del MUA, son:

$$
\begin{gathered}
v_{f}=v_{0}+a t . \\
x=v_{0} t+a t^{2} / 2 \\
v_{f}^{2}=v_{0}^{2}+2 a x .
\end{gathered}
$$

Ahora bien, hay algunos resultados relevantes que son los llamados teoremas físicos. Uno de ellos, al que resulta indispensable hacer mención, es el teorema de la velocidad media (promedio), el cual se enuncia como sigue: 
"Un cuerpo uniformemente acelerado recorre, en un determinado, intervalo de tiempo, la misma distancia que sería recorrida por un cuerpo que se desplazara a velocidad constante e igual a la velocidad media del primero, $v_{m}=\left(v_{o}+v_{f}\right) / 2 "$.

Se puede hacer notar la equivalencia de la distancia recorrida por el cuerpo en aceleración uniforme, con la distancia recorrida por el mismo cuerpo a una velocidad constante dada precisamente por su velocidad media, que es el promedio de las velocidades inicial y final.

De este teorema se desprende el resultado para el desplazamiento del cuerpo, $x=t\left(v_{o}+v_{f}\right) / 2$, lo que significa que el movimiento se comporta como rectilíneo uniforme (MRU) con una velocidad constante dada precisamente como la velocidad promedio, $v_{m}=\left(v_{o}+v_{f}\right) / 2$. En forma tal que del teorema anterior se puede desprender el siguiente enunciado,

"Un movimiento uniformemente acelerado (MUA), se comporta como un movimiento rectilíneo uniforme (MRU), $x=t\left(v_{o}+v_{f}\right) / 2$, en el entendido de que la velocidad promedio es una constante en todo el movimiento".

La característica de ser constante la velocidad promedio, es tan relevante que sin ella, no podría darse la equivalencia de un sistema uniformemente acelerado a un sistema rectilíneo uniforme, lo que es posible expresar como $x=v_{m} t$.

El teorema de la velocidad media, $x=t\left(v_{o}+v_{f}\right) / 2$, se puede justificar mediante el siguiente desarrollo:

Para el movimiento uniformemente acelerado, se tiene que la velocidad final es

$$
v_{f}=v_{0}+a t .
$$

De manera que si se calcula la velocidad media (promedio), se tiene que:

$$
v_{m}=\left(v_{o}+v_{f}\right) / 2=\left(v_{0}+v_{0}+a t\right) / 2=v_{0}+a t / 2 .
$$

De la expresión para el desplazamiento,

$$
x=v_{0} t+a t^{2} / 2=\left(v_{0}+a t / 2\right) t,
$$

se obtiene el teorema de la velocidad media,

$$
x=v_{m} t \text {. }
$$

En tal caso, cuando en la didáctica de la física, se quiere manifestar la relevancia conceptual de este teorema, es necesario diseñar situaciones de aprendizaje donde se muestre su aplicación, explicitando la transición de un MUA a un MRU, vía la presencia de una velocidad promedio. 


\section{Sistemas Promediales En La Dinámica}

En el caso de la máquina de Atwood, la tensión en la cuerda, está expresada de la forma,

$$
T=\left(2 m_{1} m_{2} /\left(m_{1}+m_{2}\right)\right) g .
$$

Cuando se llega a este resultado pareciera que no hay nada más que añadir al estudio del comportamiento del sistema, es decir, aparentemente el estado del mismo estaría totalmente discutido, pero realmente no es así, dado que adicionalmente se puede identificar una masa promedio, en el que intervienen las dos masas, $m_{1}$ y $m_{2}$, siendo el promedio expresado como una media armónica de las mismas, esto es,

$$
M=2 m_{1} m_{2} /\left(m_{1}+m_{2}\right) \text {. }
$$

De donde, se tiene que,

$$
T=M g \text {. }
$$

Las dos variables dinámicas que son la fuerza de tensión $T$ y la aceleración de la gravedad g, aparecen en una relación de proporcionalidad directa, siendo la masa equivalente $M$, la que interviene como la llamada constante de proporcionalidad. En el caso en que $m_{1}=m_{2}$, la tensión en la cuerda se reduce a $T=m_{1} g$ y por supuesto hay equilibrio en el sistema.

Vale la pena resaltar una interpretación geométrica de la media armónica, que es la referida a que los valores que intervienen, $a$ y $b$, representan las bases menor y mayor de un trapecio, $a<b$, de tal manera que al trazar las dos diagonales del trapecio, la altura que pasa por el punto de intersección resulta ser precisamente la media armónica $2 a b /(a+b)$, lo que permite identificarla como el valor más cercano al valor de $a$.

De la anterior interpretación geométrica se desprende que en el caso de la máquina de Atwood, la masa equivalente $M=2 m_{1} m_{2} /\left(m_{1}+m_{2}\right)$, tiene un valor cercano a la masa $m_{1}$, considerando que $m_{1}<m_{2}$. Tal interpretación podría ser incorporada como un argumento en una clase teórica y en todo caso es de fácil comprobación en el laboratorio.

Otro contexto de uso, referido a la cinemática, en donde aparece la media armónica, es un problema típico planteado como el hecho de que una misma distancia $d$, es recorrida por un cuerpo con dos velocidades distintas $v_{1} \mathrm{y} v_{2}$, con $v_{1}<v_{2}$. La pregunta que se desprende es ¿cuál es la velocidad media cuando se recorre una distancia total igual a $2 d$ ?

Una respuesta que pareciera obvia es que la velocidad media está dada por la media aritmética de las dos velocidades dadas $v_{1}$ y $v_{2}$. Sin embargo, ésta es una respuesta equivocada, que se presenta con frecuencia en los estudiantes. La respuesta correcta viene dada precisamente por la media armónica de ambas velocidades, esto es,

$$
v_{m}=2 d /\left(d / v_{1}+d / v_{2}\right)=2 \mathrm{v}_{1} \mathrm{v}_{2} /\left(\mathrm{v}_{1}+\mathrm{v}_{2}\right)=\mathrm{H}\left(\mathrm{v}_{1}, \mathrm{v}_{2}\right) \text {. }
$$


Recurriendo nuevamente a la interpretación geométrica, dicha velocidad promedio tiene un valor cercano a la velocidad más pequeña, $v_{l}$.

En los casos anteriormente mencionados, se hace explícita la articulación conceptual entre la matemática y la física, siendo algún tipo de promedio, su eje de articulación. Adicionalmente, se puede hacer notar el hecho de que analizar un sistema, desde la perspectiva promedial, posibilita el incorporar nuevos argumentos que pueden permitir el entender mejor y de manera más amplia, diferentes aspectos acerca del funcionamiento del sistema físico.

\section{Sistemas Promediales En Electromagnetismo}

Otra parte de la física donde aparecen herramientas promediales al encontrar un sistema equivalente es en electromagnetismo, al calcular la resistencia (capacitor) equivalente cuando se conectan dos resistencias (capacitores) en serie o en paralelo.

\section{Resistencias}

Cuando tenemos dos resistencias en conectadas en paralelo, la resistencia equivalente es (Giancoli, 2015, Serway \& Jewett, 2014, Halliday, Resnick \& Walker, 2013),

$$
R_{e}=R_{1} R_{2} /\left(R_{1}+R_{2}\right)
$$

es precisamente la mitad de la media armónica de $\mathrm{R}_{1}$ y $\mathrm{R}_{2}$, esto es,

$$
H\left(R_{1}, R_{2}\right)=2 R_{e} \text {. }
$$

El sistema original tiene un sistema promedial equivalente, dado que aparece la relación básica del sistema, ley de $\mathrm{Ohm}, V=R I$, expresada en términos de la resistencia equivalente,

$$
V=\left(H\left(R_{1}, R_{2}\right) / 2\right) I
$$

Las dos variables dinámicas que son el voltaje $V$ y la intensidad de corriente $I$, aparecen en una relación de proporcionalidad directa, siendo la resistencia equivalente $H\left(R_{1}, R_{2}\right) / 2$, la que interviene como la llamada constante de proporcionalidad.

Ahora bien, cuando se tienen dos resistencias conectadas en serie, la resistencia equivalente (Giancoli, 2015, Serway \& Jewett, 2014, Halliday, Resnick \& Walker, 2013) es:

$$
R=R_{1}+R_{2} \text {, }
$$

que es precisamente dos veces la media aritmética de $\mathrm{R}_{1}$ y $\mathrm{R}_{2}$, esto es,

$$
M\left(R_{1}, R_{2}\right)=R / 2 \text {. }
$$

\section{Capacitores}

Cuando se tienen dos capacitores conectados en serie, el capacitor equivalente es (Giancoli, 2015, Serway \& Jewett, 2014, Halliday, Resnick \& Walker, 2013), 


$$
C_{e}=C_{1} C_{2} /\left(C_{1}+C_{2}\right) \text {, }
$$

que es precisamente la mitad de la media armónica de $C_{1}$ y $C_{2}$, el valor de la capacitancia equivalente es,

$$
H\left(C_{1}, C_{2}\right)=2 C_{e}
$$

Nuevamente el sistema original tiene un sistema promedial equivalente, dado que aparece la relación básica del sistema expresado en términos de,

$$
Q=H\left(C_{1}, C_{2}\right) / \text {. }
$$

Las dos variables dinámicas que son la carga $Q$ y el voltaje $V$, aparecen en una relación de proporcionalidad directa, siendo la capacitancia equivalente $H\left(C_{1}, C_{2}\right) / 2$, la que interviene como la llamada constante de proporcionalidad Al tener dos capacitores conectados en paralelo, el capacitor equivalente (Giancoli, 2015, Serway \& Jewett, 2014, Halliday, Resnick \& Walker, 2013) es,

$$
C_{e}=C_{1}+C_{2}
$$

que es precisamente dos veces la media aritmética de $C_{1}$ y $C_{2}$ el valor,

$$
M\left(C_{1}, C_{2}\right)=C_{e} / 2 \text {. }
$$

Es de resaltarse que en cada uno de los contextos antes analizados, aparece un sistema equivalente de tipo promedial, al cual se llega vía la participación de la media aritmética ó de la media armónica, según sea el caso.

Podemos cerrar este apartado con la siguiente consideración, como lo señala Pecharromán (2013), es conveniente reconocer el proceso histórico, social y cultural de creación, desarrollo y definición, de los conceptos matemáticos, pero también de su incidencia, como es el caso de los promedios, en el contexto de la física, precisamente por el enriquecimiento conceptual en su aprendizaje. Por otra parte, la matemática, en toda su historia, se ha manifestado como un instrumento eficaz para interpretar, organizar y representar el mundo físico, que se presenta como un contexto que inspira la creación de objetos matemáticos para su estudio y aplicación.

Además, el interés racional por conocer las propiedades, relaciones y potencialidades de los objetos matemáticos creados también motiva el desarrollo de la matemática por sí misma. En definitiva, los objetos matemáticos ofrecen a la razón otro contexto que analizar, organizar e interpretar al mundo físico.

\section{Sistema Promedial Referido A La Colisión De Dos Cuerpos}

Otro caso de un sistema promedial, que por sus características especiales conviene analizar, es el que corresponde al problema de la colisión elástica de dos cuerpos de masas, $\mathrm{m}_{1}$ y $\mathrm{m}_{2}$, en una dimensión y cuando las fuerzas externas son cero, en cuyo caso la energía potencial es 
cero, por tanto la energía total es puramente la energía cinética, de modo tal que la conservación de la energía total, es equivalente a la conservación de la energía cinética.

Se parte de la consideración de que se conserva la cantidad de movimiento,

$$
\begin{gathered}
m_{1} v_{1 i}+m_{2} v_{2 i}=m_{1} v_{1 f}+m_{2} v_{2 f} \\
\text { Lo que es equivalente a, } \\
m_{1} v_{1 i}-m_{1} v_{l f}=m_{2} v_{2 f}-m_{2} v_{2 i} \\
\text { O bien, } \\
m_{1}\left(v_{1 i}-v_{l f}\right)=m_{2}\left(v_{2 f}-v_{2 i}\right) .
\end{gathered}
$$

Cuando la velocidad del centro de masa permanece constante, se cumple que,

$$
V_{C M}=\left(m_{1} v_{1}+m_{2} v_{2}\right) /\left(m_{1}+m_{2}\right) \text {. }
$$

Esta velocidad es una media aritmética ponderada, donde las variables son las velocidades $v_{1} \mathrm{y} v_{2}$, y las ponderaciones son las masas, $m_{1} \mathrm{y} m_{2}$.

La energía cinética se conserva en el choque de dos cuerpos, esto es,

$$
\begin{gathered}
1 / 2 m_{1} v_{1 i}^{2}+1 / 2 m_{2} v_{2 i}^{2}=1 / 2 m_{1} v_{1 f}^{2}+1 / 2 m_{2} v_{2 f .}^{2} \\
\text { De donde, } \\
1 / 2 m_{1}\left(v^{2}{ }_{1 i}-v_{1 f}^{2}\right)=1 / 2 m_{2}\left(v^{2} 2 f-v^{2}{ }_{2 i}\right) . \\
\text { Realizando algunas operaciones, } \\
m_{1}\left(v_{1 i}-v_{l f}\right)\left(v_{l i}+v_{l f}\right) / 2=m_{2}\left(v_{2 f}-v_{2 i}\right)\left(v_{2 i}+v_{2 f}\right) / 2 .
\end{gathered}
$$

De manera tal, que de la conservación de la cantidad de movimiento se cumple,

$$
m_{1}\left(v_{1 i}-v_{1 f}\right)=m_{2}\left(v_{2 f}-v_{2 i}\right) .
$$

Se obtiene así el resultado para las velocidades medias (promedio) de los dos cuerpos,

$$
\left(v_{1 i}+v_{1 f}\right) / 2=\left(v_{2 i}+v_{2 f}\right) / 2 .
$$

Lo que nos dice que, las velocidades promedio (medias aritméticas) de cada uno de los cuerpos se conservan.

Por su relevancia este resultado se puede considerar como un teorema promedial de la física,

$$
V\left(v_{1 i}, v_{l f}\right)=V\left(v_{2 i}, v_{2 f}\right)
$$

El hecho de que las velocidades promedio de ambos cuerpos se conserven, proviene a su vez de las condiciones previas de conservación, tanto de la cantidad de movimiento como de la energía cinética, sin las 
cuales no se cumpliría tal teorema. Es de interés resaltar que el resultado anterior, es independiente de las masas de los dos cuerpos que chocan.

De los desarrollos anteriores se desprende que hay una cierta forma de equivalencia de los tres teoremas: conservación de la cantidad de movimiento, conservación de la energía cinética y la conservación de las velocidades promedios, bajo el entendido de que si dos de ellos de cumplen, entonces el tercero se cumplirá. En todo caso la característica promedial del sistema es lo que propicia que lo anterior se pueda ocurrir, es decir, si en un sistema dado, dos de las propiedades se cumplen, la tercera propiedad siempre se cumplirá.

En este sistema del choque elástico de dos cuerpos, lo promedial aparece en el caso de la velocidad del centro de masa y bajo la conservación de la energía cinética y de la cantidad de movimiento, se presenta el resultado promedial de la igualdad o conservación de las velocidades promedio de ambos cuerpos, mismo que se puede considerar como el sistema promedial equivalente.

Los tres casos posibles que se pueden presentar, respecto a las relaciones existentes entre las leyes de conservación son los siguientes:

i) Conservación de energía cinética + conservación de momento lineal $\Rightarrow$ conservación de velocidades promedio.

ii) Conservación de energía cinética + conservación de velocidades promedio $\Rightarrow$ conservación de momento lineal.

iii) Conservación de momento lineal + conservación de velocidades promedio $\Rightarrow$ conservación de energía cinética.

Considerando los casos anteriores, se identifica una relación entre dos leyes de conservación de la física y otra de carácter promedial, lo que lleva a plantear la pregunta ¿será acaso que las dos primeras, se puedan expresar en forma promedial?, en la siguiente sección se abordará esta cuestión.

\section{Otro Enfoque Promedial En La Colisión Elástica De Dos Cuerpos}

Cuando en un sistema dado, como es el caso del choque elástico de dos cuerpos, se manifiesta la conservación de la energía cinética y la conservación de la cantidad de movimiento. Ahora bien, desde la perspectiva de lo promedial, ambas leyes se pueden reinterpretar en términos de medias ponderadas, donde las ponderaciones están dadas por las respectivas masas de las partículas, esto es, para la energía cinética,

$$
1 / 2 m_{1} v_{1 i}^{2}+1 / 2 m_{2} v_{2 i}^{2}=1 / 2 m_{1} v_{1 f}^{2}+1 / 2 m_{2} v_{2 f}^{2}
$$

Es de hacerse notar que al realizar la simple operación de dividir entre la suma de las masas,

$$
1 / 2\left(m_{1} v_{1 i}^{2}+m_{2} v_{2 i}^{2}\right) /\left(m_{1}+m_{2}\right)=1 / 2\left(m_{1} v_{1 f}^{2}+m_{2} v_{2 f}^{2}\right) /\left(m_{1}+m_{2}\right) .
$$


Tal conservación se puede interpretar como el hecho de que las medias aritméticas ponderadas $M_{p}$, de los cuadrados de las velocidades iniciales y finales de ambas partículas, son iguales, es decir,

$$
M_{p}\left(v^{2}{ }_{1 i}, v^{2}{ }_{2 i} ; m_{1}, m_{2}\right)=M_{p}\left(v^{2}{ }_{1 f}, v^{2}{ }_{2 f} ; m_{1}, m_{2}\right) .
$$

De igual forma, en el caso de la conservación de la cantidad de movimiento,

$$
m_{1} v_{1 i}+m_{2} v_{2 i}=m_{1} v_{1 f}+m_{2} v_{2 f}
$$

dividiendo entre la suma de las masas,

$$
\left(m_{1} v_{l i}+m_{2} v_{2 i}\right) /\left(m_{1}+m_{2}\right)=\left(m_{1} v_{l f}+m_{2} v_{2 f}\right) /\left(m_{1}+m_{2}\right) .
$$

Se obtiene el resultado que se expresa en términos de una la igualdad (conservación) entre los promedios ponderados de las velocidades iniciales y finales de ambas partículas, esto es,

$$
M_{p}\left(v_{1 i}, v_{2 i} ; m_{1}, m_{2}\right)=M_{p}\left(v_{1 f}, v_{2 f} ; m_{1}, m_{2}\right) .
$$

Es posible señalar que las dos leyes de conservación de la energía cinética y de la cantidad de movimiento, desde la perspectiva de lo promedial, se pueden reinterpretar como la conservación (igualdad) entre las medias ponderadas de los cuadrados de las velocidades y las medias ponderadas de las velocidades iniciales y finales, respectivas de las dos partículas, que intervienen en el choque elástico. Todo lo cual lleva a incorporar en el discurso didáctico de la enseñanza de la física, un elemento conceptual, que posibilita el estructurar un argumento adicional, en este caso sustentado en lo promedial.

Podemos entonces enunciar los siguientes teoremas promediales:

i) Un sistema físico (dinámico) en donde la fuerza total es cero, que cumple con las leyes de conservación de la cantidad de movimiento y de la energía cinética, puede considerarse como un sistema promedial, en el que se conservan las medias aritméticas ponderadas de las velocidades iniciales y finales, cuyas ponderaciones están dadas por las masas de los cuerpos.

ii) Un sistema físico (dinámico) en donde la fuerza total es cero, que cumple con las leyes de conservación de la cantidad de movimiento y de la energía cinética, puede considerarse como un sistema promedial, en el que se conservan las medias 
aritméticas ponderadas de los cuadrados de las velocidades iniciales y finales cuyas ponderaciones están dadas por las masas de los cuerpos.

No deja de ser sorprendente que dos resultados importantes de la física, como son las leyes de conservación de la energía cinética y de la cantidad de movimiento, puedan ambas ser expresadas en términos de teoremas promediales, referidos a los respectivos promedios ponderados, siendo las masas las que intervienen en sus ponderaciones. En tal caso, vale preguntarse ¿qué efectos conceptuales sobre la física tiene el incorporar a su discurso argumentativo la perspectiva promedial, dada por los teoremas antes enunciados?

Como lo expresa Pecharromán (2013), al incorporar otra representación, la información es distinta, aunque requiere mantener invariantes sus propiedades, y posiblemente en detrimento de otras representaciones establecidas o ya conocidas previamente. Adicionalmente menciona, en definitiva, los conceptos matemáticos tienen un proceso histórico, social y cultural de creación, desarrollo y definición. En cada momento de la historia, existe un desarrollo social y cultural que moldea, condiciona y determina el conocimiento que se considera válido o posible, desde un conocimiento previo aceptado y las nuevas perspectivas que puedan considerarse. De manera tal que en nuestro caso, al incorporar en algunos sistemas físicos la representación promedial, aparece una nueva perspectiva que sin duda propicia un enriquecimiento conceptual que no se queda en la matemática sino que trasciende a la física, todo lo cual contribuye a mostrar evidencias de la articulación conceptual de ambas ciencias.

\section{El Problema De Los Dos Cuerpos}

En los cursos de Mecánica Clásica, que se imparten en la segunda mitad de una licenciatura en Física, se aborda el Problema de los Dos Cuerpos, Goldstein (2001), por su gran importancia teórica, en este caso lo trataremos con el enfoque Lagrangiano. En el mismo intervienen dos masas $\mathrm{m}_{1} \mathrm{y} \mathrm{m}_{2}$, con sus respectivos vectores de posición $\boldsymbol{r}_{\boldsymbol{1}}, \boldsymbol{r}_{2}$, respecto a un origen común. Un concepto importante que interviene en este problema es el centro de masa $(\mathrm{CM})$, el cual tiene a su vez asociado un vector de posición $\boldsymbol{R}$.

De tal manera que la Lagrangiana del sistema es:

$$
L=T-U \text {. }
$$

Siendo $T$ la energía cinética y $U$ la energía potencial del sistema:

$$
L=1 / 2 m_{1} v_{1}^{2}+1 / 2 m_{2} v_{2}^{2}-U\left(\left|\boldsymbol{r}_{2}-\boldsymbol{r}_{1}\right|\right) \text {. }
$$




$$
\begin{aligned}
& \text { Definiendo, } \\
& \boldsymbol{r}=\boldsymbol{r}_{2}-\boldsymbol{r}_{1}, \\
& \boldsymbol{R}\left(\boldsymbol{r}_{1}, \boldsymbol{r}_{2}, ; m_{1}, m_{2}\right)=\left(m_{1} \boldsymbol{r}_{1}+m_{2} \boldsymbol{r}_{2}\right) /\left(m_{1}+m_{2}\right),
\end{aligned}
$$

donde $\boldsymbol{r}$, es la posición relativa de los dos cuerpos y $\boldsymbol{R}$, el vector de posición del centro de masa.

Sustituyendo y realizando operaciones, la Lagrangiana queda expresada como:

$$
L=1 / 2\left(m_{1}+m_{2}\right) V_{R}^{2}+1 / 2 m_{1} m_{2} /\left(m_{1}+m_{2}\right) V_{r}^{2}-U(\boldsymbol{r}) .
$$

Siendo, $V_{R}$ y $M_{T}=m_{1}+m_{2}$, la velocidad del CM y la masa total del sistema; además $\mathrm{V}_{\mathrm{r}}$, es la velocidad de la posición relativa. Note que,

$$
M_{T}=2 X\left(m_{1}, m_{2}\right) .
$$

En el tratamiento teórico que presenta Goldstein (2001), después de realizar lo anterior, "define" a la masa reducida $\mu$ como,

$$
\begin{gathered}
1 / \mu=1 / m_{1}+1 / m_{2}, \\
\text { de donde, } \\
\mu=m_{1} m_{2} /\left(m_{1}+m_{2}\right),
\end{gathered}
$$

aunque no se menciona que tal masa reducida es la mitad de la media armónica de las dos masas, esto es,

$$
H\left(m_{1}, m_{2}\right)=2 \mu .
$$

Como se sabe el resultado general de las medias armónica, geométrica y aritmética para dos valores dados es, $H \leq G \leq X$, se cumple que,

$$
H\left(m_{1}, m_{2}\right) / 2 \leq X\left(m_{1}, m_{2}\right) / 2<2 X\left(m_{1}, m_{2}\right) \text {. }
$$

Esto quiere decir que la masa reducida $\mu=m_{1} m_{2} /\left(m_{1}+m_{2}\right)$, es menor que la masa total,

$$
\begin{gathered}
\mu<2 X\left(m_{1}, m_{2}\right) . \\
\mu<M_{T} .
\end{gathered}
$$

Por supuesto, este último resultado no es resaltado en los libros que tratan acerca del estudio de los sistemas de dos cuerpos, tales como Goldstein (2001) y otros.

En el problema de dos cuerpos, visto como un sistema promedial, cabe resaltar la participación que tienen las masas de los cuerpos (variables pasivas), tanto en la masa total del sistema, como en la masa reducida. Por otra parte, en lo que se refiere a las variables dinámicas, vector de posición y velocidad del CM, en su definición aparecen promedios ponderados, cuyas 
ponderaciones son las propias masas. De manera tal que, los promedios permiten resolver el problema de los dos cuerpos, que como se sabe es un problema resuelto, sin embargo, el tratamiento promedial presentado vislumbra una nueva faceta que abre otras perspectivas hacia problemas de más cuerpos.

\section{Conclusiones}

De lo anteriormente analizado, se desprende que en el análisis de sistemas de dos elementos, donde exista su sistema equivalente, siempre aparece alguna forma de promedio matemático involucrado para lograr tal equivalencia. Por ejemplo, en el caso del problema de los dos cuerpos, se parte de considerar el vector de posición del centro de masa, como una media ponderada (de ambas masas). Resulta, que sin ésta condición, no podría llevarse a un sistema equivalente.

Cabe señalar que en este trabajo sólo se han presentado unos pocos casos donde tienen presencia los promedios, aunque queda entendido de que los sistemas promediales tienen una participación amplia en el estudio de la física.

En el choque de dos cuerpos, se considera que es un sistema aislado (fuerzas externas cero por lo tanto potencial de interacción cero). Por lo mostrado en este trabajo, este sistema llegará al equilibrio a través de promedios, ya que en este caso particular las leyes de la física (conservación del momento lineal y energía) se pueden reescribir en términos de promedios. Así pues, existe una relación cercana entre el equilibrio y los promedios.

Es conveniente remarcar, la relevancia de los promedios en la didáctica de la física, aunque en ocasiones, su aparente obviedad lleva a omitir su trascendencia conceptual y por tanto a una exclusión inapropiada para propiciar en los estudiantes, aprendizajes más completos de los sistemas físicos que por las características descritas son de tipo promedial, lo que puede permitir explorar y entender mejor el mundo físico.

Los sistemas promediales son un claro ejemplo de la necesaria articulación conceptual entre la matemática y la física, misma que requiere ser explicitada en la enseñanza de ambas ciencias.

Las leyes de conservación de la cantidad de movimieno y de la energía cinética, se pueden reinterpretar como la conservación de un promedio ponderado de las velocidades y de los cuadrados de las velocidades de las parículas, siendo las masas las correspondientes ponderaciones.

La matemática, en toda su historia, se ha manifestado como un instrumento eficaz para interpretar, organizar y representar el mundo físico, y recíprocamente, el mundo sensible se presenta como un contexto que inspira la creación de objetos matemáticos. Además, el interés racional por conocer 
las propiedades, relaciones y potencialidades de los objetos matemáticos creados motiva el desarrollo de la matemática y en muchas ocasiones de la misma física.

En lo que se refiere a la didáctica de la física, el estudiar a los sistemas promediales, posibilita el tener una representación complementaria, lo que puede permitir a los estudiantes el identificar una conceptualización más amplia de los problemas involucrados, es decir, habrá una cierta ganancia cognitiva, en el sentido de incorporar otra forma conceptual de entender al sistema original.

\section{References:}

1. Almudí, J., Zuza, K., \& Guisasola, J. (2016). Aprendizaje de la teoría de inducción electromagnética en cursos universitarios de física general. Una enseñanza por resolución guiada de problemas. La Enseñanza de las Ciencias , 34 (2), 7-24.

2. Alonso, M. , Finn, E. J.(1967). Física Volumen I : Mecánica. Estados Unidos : Addison- Wesley Iberoamericana.

3. Bachelard, G. (2000). La formación del espíritu científico (23 ed., trad. José Babini). México: Siglo XXI.

4. Bertalanffy, L. (1989). Teoría general de los sistemas. Fondo de cultura económica. México.

5. Catalina, A. F. (2013). La búsqueda de analogías y diferencias como estrategia de enseñanza de las interacciones a distancia y del concepto de campo en Física. La Enseñanza de las Ciencias , 31 (3), 280-281.

6. Fagundez, T., \& Castells, M. (2012). La argumentación en clases universitarias de física: Una perspectiva retórica. La Enseñanza de las Ciencias , 30 (2), 153-174.

7. Flores, A. P. (2009). Las medias como promedios ponderados. Miscelánea Matemática. México.

8. Garcia-Colín, L. (1970). Introducción a la termodinámica clásica. México: Trillas.

9. Giancoli, D. (2015). Physics: Principles with Applications with Mastering Physics. Estados Unidos: Pearson Education

10. Goldstein, H., Poole, C. \& Safko, J. (2001). Classical Mechanics. Estados Unidos: Pearson Education.

11. Halliday, D., Resnick, R. Walker, J. (2013). Fundamentals of Physics (10th. ed.). Estados Unidos: Wiley.

12. Mederos, O., \& Martínez, J. (2014). La resolución de problemas y la formación y desarrollo de conceptos. El concepto de media aritmética. (U. d. Andes, Ed.) Números. Revista de Didáctica de las Matemáticas , 53-63. 
13. Ortíz, P. ( 2009). Tratados II. Sobre el equilibrio de las figuras planas Editorial Gredos, España.

14. Pecharromán, C. Naturaleza de los objetos matemáticos: representación y significado. Enseñanza de las Ciencias NÚM. 31.3 (2013): 121-134.

15. Pocoví, C., \& Collivadino, C. (2014). Traducción entre lenguajes simbólicos de distintas áreas del conocimiento: el caso del flujo del campo eléctrico. La Enseñanza de las Ciencias , 32 (2), 53-69.

16. Rondero, C., Reyes, A., Acosta, J. A. (2015). Aspectos Históricos del Cálculo de Leibniz: Incidencia y Aplicación en la Didáctica de las Matemáticas. Revista Números, España.

17. Rittaud, B. (2009). Qué irracional. El fabuloso destino de $\sqrt{ } 2$. Consejo Nacional para la Cultura y las Artes. México.

18. Sales, E. T., Freire, O. J., \& María, L. G. (2015). La enseñanza de la gravitación universal de Newton orientada por la historia y filosofía de la ciencia: una propuesta didáctica con un enfoque en la argumentación. La Enseñanza de las Ciencias , 33 (1), 205-223

19. Serway, R. A. \& Jewett, J. (2014). Physics for scientists and Engineers (9th. ed.). Estados Unidos: Wadsworth Publishing Inc.

20. Sinarcas, V., \& Solbes, J. (2013). Dificultades en el aprendizaje y la enseñanza de la Física Cuántica en el bachillerato. La Enseñanza de las Ciencias , 31 (3), 9-25. 